\title{
Article \\ Semi-Rational Design of Proteus mirabilis L-Amino Acid Deaminase for Expanding Its Substrate Specificity in $\alpha$-Keto Acid Synthesis from L-Amino Acids
}

\author{
Anwen Fan ${ }^{1}$, Ziyao Wang ${ }^{1}$, Haojie $Q u^{1}$, Yao Nie ${ }^{1,2, *(D)}$ and Yan $X u^{1,3}$ \\ 1 Lab of Brewing Microbiology and Applied Enzymology, Key Laboratory of Industrial Biotechnology, \\ Ministry of Education, School of Biotechnology, Jiangnan University, Wuxi 214122, China; \\ fananwen1998@163.com (A.F.); ewqwyl123@sina.com (Z.W.); 1051200227@stu.jiangnan.edu.cn (H.Q.); \\ biosean@126.com (Y.X.) \\ 2 Suqian Industrial Technology Research Institute of Jiangnan University, Suqian 223814, China \\ 3 State Key Laboratory of Food Science and Technology, Jiangnan University, Wuxi 214122, China \\ * Correspondence: ynie@jiangnan.edu.cn; Tel.: +86-510-8519-7760
}

Citation: Fan, A.; Wang, Z.; Qu, H.; Nie, Y.; Xu, Y. Semi-Rational Design of Proteus mirabilis L-Amino Acid Deaminase for Expanding Its Substrate Specificity in $\alpha$-Keto Acid Synthesis from L-Amino Acids. Catalysts 2022, 12, 175. https:// doi.org/10.3390/catal12020175

Academic Editor: Francesco Secundo

Received: 2 January 2022

Accepted: 24 January 2022

Published: 29 January 2022

Publisher's Note: MDPI stays neutral with regard to jurisdictional claims in published maps and institutional affiliations.

Copyright: () 2022 by the authors Licensee MDPI, Basel, Switzerland. This article is an open access article distributed under the terms and conditions of the Creative Commons Attribution (CC BY) license (https:// creativecommons.org/licenses/by/ $4.0 /)$.

\begin{abstract}
L-amino acid deaminases (LAADs) are flavoenzymes that catalyze the stereospecific oxidative deamination of L-amino acids into $\alpha$-keto acids, which are widely used in the pharmaceutical, food, chemical, and cosmetic industries. However, the substrate specificity of available LAADs is limited, and most substrates are concentrated on several bulky or basic L-amino acids. In this study, we employed a LAAD from Proteus mirabilis (PmiLAAD) and broadened its substrate specificity using a semi-rational design strategy. Molecular docking and alanine scanning identified F96, Q278, and E417 as key residues around the substrate-binding pocket of PmiLAAD. Site-directed saturation mutagenesis identified E417 as the key site for substrate specificity expansion. Expansion of the substrate channel with mutations of E417 (E417L, E417A) improved activity toward the bulky substrate L-Trp, and mutation of E417 to basic amino acids (E417K, E417H, E417R) enhanced the universal activity toward various L-amino acid substrates. The variant PmiLAAD ${ }^{\mathrm{E} 417 \mathrm{~K}}$ showed remarkable catalytic activity improvement on seven substrates (L-Ala, L-Asp, L-Ile, L-Leu, L-Phe, L-Trp, and L-Val). The catalytic efficiency improvement obtained by E417 mutation may be attributed to the expansion of the entrance channel and its electrostatic interactions. These PmiLAAD variants with a broadened substrate spectrum can extend the application potential of LAADs.
\end{abstract}

Keywords: L-amino deaminase; amino acids; keto acids; substrate spectrum; substrate channel

\section{Introduction}

L-amino acid deaminases (LAADs) are flavoenzymes containing non-covalently bound flavin adenine dinucleotide, which catalyzes the stereospecific oxidative deamination of L-amino acids into $\alpha$-keto acids and ammonia [1] (Figure 1).<smiles>[X]C[C@H](N)C(=O)O</smiles>

L-amino acid

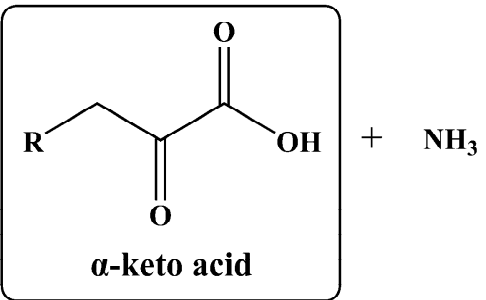

Figure 1. Oxidative deamination of L-amino acids catalyzed by L-amino acid deaminases.

A LAAD is an advantageous and suitable catalyst for producing keto acids, because it can synthesize keto acids in whole cells without the addition of a cofactor or the generation of potentially harmful by-products (e.g., hydrogen peroxide) compared with other 
enzymes that can produce keto acids, such as L-amino acid oxidase and aminotransferase [2]. Keto acids are organic acids containing a carboxyl acid group and a ketone group, which are widely used in the pharmaceutical, food, chemical, and cosmetics industries [3]. Phenylpyruvic acids are used for the synthesis of D-phenylalanine [4] and phenyllactic acid, which are applied in medicine $[5,6] . \alpha$-Keto- $\gamma$-methylthiobutyric acids play an important role in the therapy to limit tumor growth [7] and are used as methionine supplements in livestock feed [8]. Pyruvic acids are utilized in synthesizing vitamins, cereal protection agents, and pesticides $[9,10] . \alpha$-Ketoisovaleric acids stimulate muscle growth in livestock [11] and reduce renal filtration pressure [12].

Thus, the production of keto acids by LAADs has attracted substantial research attention in recent years. To date, various keto acids have been successfully produced through bioconversion by LAADs [13-15]. In addition, LAADs are widely used in multienzyme cascade systems to produce expensive D-amino acids, which have diverse applications in the pharmaceutical, agricultural, food, and cosmetics industries $[5,16,17]$. Parmeggiani et al. [18] designed a novel one-pot multi-enzyme method employing LAAD, phenylalanine ammonia lyase, and borane-ammonia to synthesize substituted D-Phe from cheap cinnamic acid. The same group developed a three-enzyme system to convert Larylalanines to D-arylalanines, which combined LAAD and D-amino acid dehydrogenase with glucose dehydrogenase for cofactor recycling [19]. Zhang et al. [20] also successfully synthesized optically pure D-Phe from L-Phe using a similar system. Moreover, Walton et al. [21] coupled LAADs with aminotransferase to produce a range of D-Phe derivatives. Considering the wide application of LAADs in keto acid synthesis and multienzyme system construction, it is necessary to expand their substrate specificity because a LAAD mostly exhibits specificity toward bulky amino acids and basic amino acids [2].

Thus, there has been extensive research effort toward engineering LAADs for increased catalytic ability. Wu et al. [22] generated mutations on four essential residues (F93S/P186A/M394V/F184S) of PmiLAAD, successfully achieving a 6.6-fold higher specific activity towards L-Phe compared with that of the wild-type. Song et al. [23] engineered PvLAAD to obtain a higher L-Leu conversion rate $(94.25 \%)$ by optimizing the plasmid origin with different copy numbers, modulating messenger RNA structure downstream of the initiation codon, and designing the sequences at the ribosome binding site. Hossain et al. [15] have constructed a double mutant (K104/A337) of PvLAAD through error-prone polymerase chain reaction (erPCR), and a 1.3-fold activity towards L-Met of the wild-type was achieved. Similar studies have been aimed at enhancing the activity of LAADs toward substrates such as L-Val [24] and L-1-naphthylalanine [25]. However, the metabolic or protein engineering of LAADs, including the examples mentioned above, has mostly focused on improving the activity against a certain substrate, whereas research on the development of a single LAAD catalyst capable of producing various keto acids is limited.

To address this gap, in this study, we developed new PmiLAAD variants with higher activity toward various types of L-amino acids via a semi-rational design strategy. PmiLAAD was modeled using the SWISS MODEL server. The docking conformations of seven L-amino acids into the substrate-binding pocket of PmiLAAD were analyzed, and key residues were identified for site-directed saturation mutagenesis after alanine scanning of the relevant sites around the pocket. We then constructed PmiLAAD variants with broader substrate specificity compared with that of the wild-type, and the involved mechanism was analyzed by comparing the substrate entrance channel conformation between the wild-type and the variant with enhanced catalytic ability.

\section{Results and Discussion}

\subsection{Homologous Modeling and Prediction of Functional Sites}

Seven L-amino acids were docked into the active site of the homologous model of PmiLAAD by AutoDock. The residues within $4 \AA$ distance from the L-amino acid ligand were determined as potential key sites for substrate binding, and the corresponding residues of each L-amino acid substrate are listed in Table 1. An example of the docking result for 
L-Trp is shown in Figure 2, which had the largest number of residues around it compared to the other substrates. The docking result with the highest score was chosen to predict the key residues. As shown in Figure 2, the residues Q99 and R315 interact with the carboxyl group of L-Trp, and the residue G437 is connected to the amino group of L-Trp, which is consistent with the residues reported to interact with the L-amino acid substrate of PvLAAD [26]. Alanine scanning was then performed on each residue to determine the key residues for mutation. The sites chosen for alanine scanning in our study were mostly different from the sites that were selected in previous studies by other researchers (Table 1). Compared with random mutations, this semi-rational strategy based on the analysis of protein structure effectively reduced the screening effort.

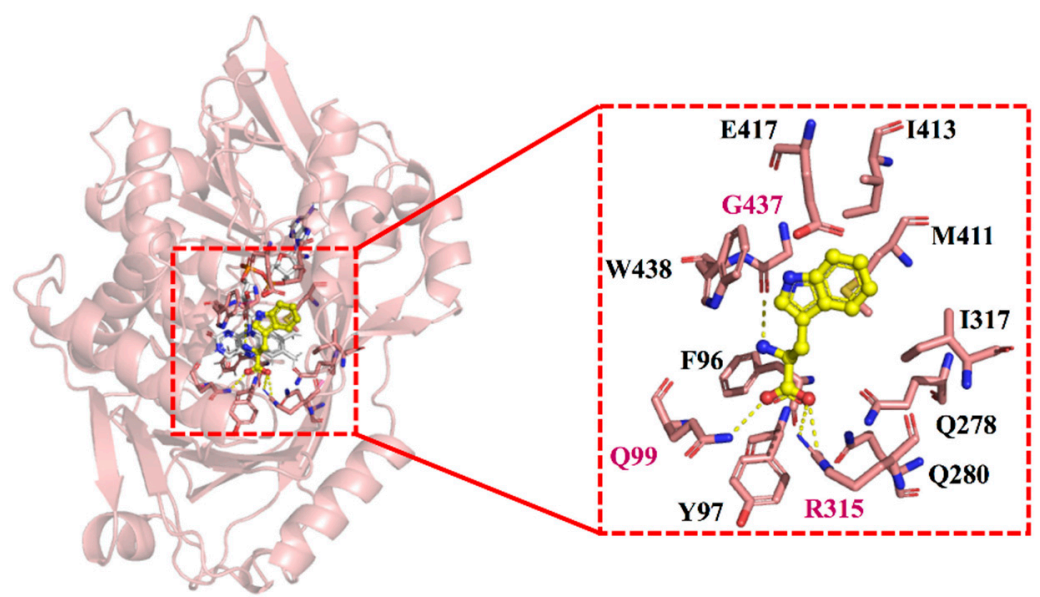

Figure 2. The docking results of L-Trp to the PmiLAAD model.

Table 1. The active sites selected in our study and in previous studies by other researchers ${ }^{\mathrm{a}}$.

\begin{tabular}{ccc}
\hline Substrate & Active Sites for Mutation & Reference \\
\hline L-Ala & M411 & This study \\
L-Val & Y97, Q278, Q280, M411 & This study \\
L-Ile & Q278, I317, M411 & This study \\
L-Leu & Q278, I317, M411, I413, W438 study \\
L-Phe & Q278, I317, M411, E417 & This study \\
L-Trp & Y97, Q99, Q278, Q280, R315, I317I, M411, I413, E417, G437, W438 & This study \\
L-Asp & F96, Y97, G98, Q99, Q278, Q280, M411, G437, W438 & This study \\
\hline L-Phe & F93, F184, P186, M394 (Proteus mirabilis) & {$[22]$} \\
L-Leu & Tuning the transcription and translation levels (Proteus vulgaris) & {$[23]$} \\
L-Met & K104, A337 (Proteus vulgaris) & {$[15]$} \\
L-Val & N100,Q276,R316,F318 (Proteus myxofaciens) & {$[24]$} \\
L-1-naphthylalanine & F318A, V412A, V438P (Proteus myxofaciens) & {$[25]$} \\
\hline
\end{tabular}

a Note: The active sites selected for alanine scanning in our study were sites within $4 \AA$ Acope of each substrate according to the docking results.

\subsection{Determination of Key Residues via Alanine Scanning}

An alanine-scanning strategy was employed to observe and compare the effects of the mutations at different sites on the activities, which helped to reduce the effort required in the processes of saturation mutagenesis and mutant screening. Alanine scanning is a method used in molecular biology and protein biochemistry to identify the molecular components that are essential for the function, form, and stability of proteins or peptides [27]. The substitution of alanine for the original amino acid residue causes all side chain atoms of a certain site to be removed, except for one functionally, largely the neutral methyl group, which usually leads to changes in protein functions. Alanine scanning was performed on all 17 residues selected by the results of molecular docking, and the activity assay 
on all of the resulting variants was conducted according to a colorimetric assay using 2,4-dinitrophenylhydrazine (DNPH).

Alanine scanning showed that the sites leading to increased activity were different for the different substrates (Figures 3 and S1). Taken as a whole, among all the variants obtained by alanine mutation, PmiLAAD ${ }^{\mathrm{F} 96 \mathrm{~A}}$ and PmiLAAD ${ }^{\mathrm{E} 417 \mathrm{~A}}$ showed activity improvement on multiple L-amino acid substrates. PmiLAAD ${ }^{\mathrm{F} 96 \mathrm{~A}}$ showed increased activity toward L-Ile (1.03-fold), L-Phe (2.71-fold), L-Trp (1.79-fold), and L-Val (1.14-fold), whereas PmiLAAD ${ }^{\text {E417A }}$ showed increased activity toward L-Ile (1.87-fold), L-Phe (5.59-fold), L-Trp (2.58-fold), and L-Val (1.33-fold). PmiLAADQ278A also showed increased activity toward L-Ala (3.28-fold), L-Leu (1.22-fold), and L-Trp (4.13-fold). In addition, residue Q278 was located within a $4 \AA$ scope around the substrate-binding site toward six of the seven L-amino acid substrates. Based on these results, F96, E417, and Q278 were selected for site-directed saturation mutagenesis in subsequent experiments.

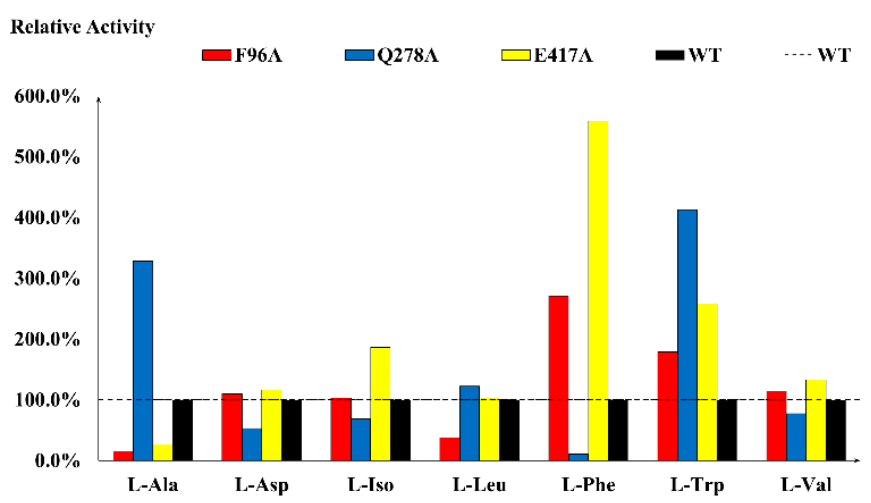

Figure 3. The results of alanine scanning on residues F96, Q278, and E417. The black dotted line indicates the relative activity (100\%) of wild-type PmiLAAD.

\subsection{Site-Directed Saturation Mutagenesis at Potential Key Residues}

To ensure the integrity and dependability of the screening results, site-directed saturation mutagenesis at the three sites was conducted on the three key residues determined by alanine scanning (F96, E417, and Q278) based on mutation primer design and homologous recombination of the PmiLAAD wild-type plasmid. Nineteen mutants at each site were constructed and expressed in Escherichia coli (DE3). The specific activity of all 57 variants of PmiLAAD was measured by the DNPH method according to the intensity of the reddish-brown color indicating different amounts of keto acids produced, which is calculated according to the corresponding standard curves. As shown in Figure 4, the catalytic properties of the seven substrates were obtained by different variants.

Among the mutations on residue F96, except for alanine, only PmiLAAD ${ }^{\mathrm{F} 96 \mathrm{~K}}$ showed increased activity (1.84-fold) toward L-Asp. Mutations of Q278, including PmiLAADQ278C, PmiLAAD ${ }^{\text {278I }}$, PmiLAAD $278 \mathrm{~L}$, and PmiLAAD ${ }^{\text {2785 }}$, resulted in enhanced activity toward L-Trp, among which PmiLAADQ278C had the highest activity (2.44-fold). Mutations at site E417 improved the catalytic activity toward diverse substrates. For example, the catalytic activity of PmiLAAD ${ }^{\mathrm{E} 417 \mathrm{H}}$ toward L-Ala, L-Asp, and L-Trp was 3.25-, 3.30-, and 2.48-fold higher than that of the wild type, respectively. The catalytic activity of PmiLAAD ${ }^{\mathrm{E} 417 \mathrm{R}}$ toward L-Ala, L-Asp, L-Phe, and L-Trp was 3.60-, 1.68-, 3.94-, and 6.10-fold higher than that of the wild type, respectively. The variant PmiLAAD ${ }^{\mathrm{E} 417 \mathrm{~S}}$ also showed increased activity toward various substrates, including L-Ala (1.63-fold), L-Asp (2.26-fold), L-Ile (1.80-fold), L-Leu (1.15-fold), and L-Trp (4.42-fold). 
A

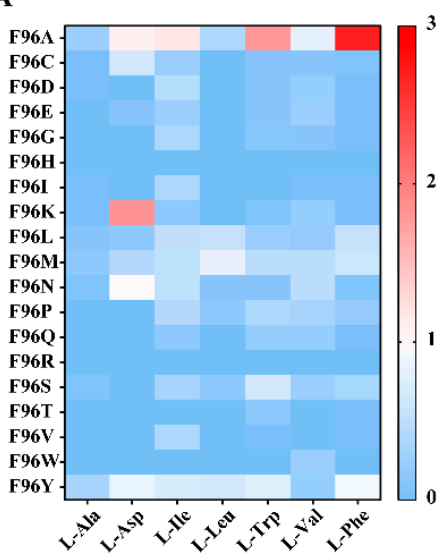

B

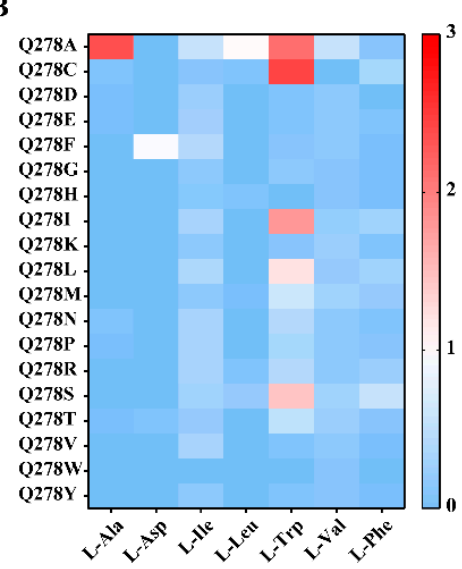

C

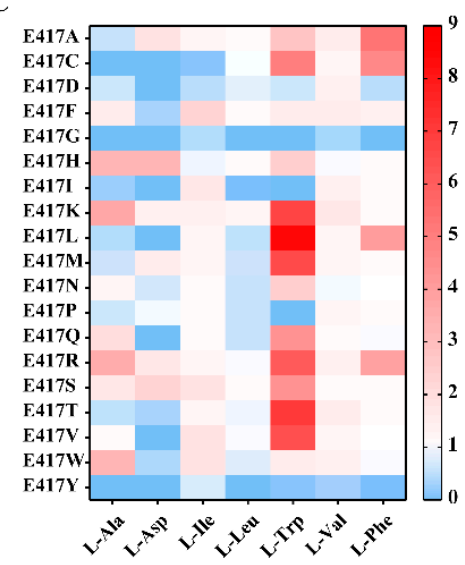

Figure 4. Heatmaps showing the relative activity of variants obtained by site-directed saturation mutagenesis toward seven L-amino acid substrates compared with that of wild-type PmiLAAD. (A) Site-directed saturation mutagenesis at site F96. (B) Site-directed saturation mutagenesis at site Q278. (C) Site-directed saturation mutagenesis at site E417. Relative activity higher and lower than that of the wild-type is shown in red and blue, respectively.

Notably, the majority of variants of PmiLAAD showing significantly increased catalytic efficiency toward the corresponding L-amino acid substrates were those harboring mutations at residue E417, such as PmiLAAD ${ }^{\text {E417A }}$, PmiLAAD $^{\text {E417H }}$, PmiLAAD ${ }^{\text {E417K, }}$ PmiLAAD $^{\text {E417R }}$, PmiLAAD ${ }^{\text {E417S }}$, and PmiLAAD ${ }^{\text {E417W }}$. On the one hand, the substrate L-Trp showed the best activity enhancement by the site-directed saturation method: the catalytic activities of PmiLAAD ${ }^{\text {E417L }}$ and PmiLAAD ${ }^{\text {E417A }}$ were 8.44-fold and 2.73-fold versus that of the PmiLAAD wild-type, respectively. On the other hand, the mutations of E417 to basic amino acid residues, such as PmiLAAD ${ }^{\text {E417K }}$, PmiLAAD ${ }^{\text {E417H }}$, and PmiLAAD ${ }^{\text {E417R }}$, exhibited obvious activity enhancement toward multiple substrates (Figure S3). PmiLAAD ${ }^{\mathrm{E} 417 \mathrm{~K}}$ was identified as the variant with the most remarkable catalytic activity improvement on all seven types of L-amino acid substrates. The activity of PmiLAAD ${ }^{\mathrm{E} 417 \mathrm{~K}}$ toward L-Ala, L-Asp, L-Ile, L-Leu, L-Phe, L-Trp, and L-Val was 3.77-fold, 1.41-fold, 1.34-fold, 1.29-fold, 1.05-fold, 6.65-fold, and 1.68-fold higher, respectively, than that of wild-type PmiLAAD (Figure S3A). As a result, the E417K mutation effectively enlarged the substrate spectrum of PmiLAAD, indicating that E417 is a promising site for improving the specific activity of PmiLAAD toward certain L-amino acids. Consequently, the catalytic ability of PmiLAAD was enhanced from a new perspective of the substrate spectrum, rather than by merely increasing the enzyme activity. The variants with broader substrate specificity obtained in our research have promising application prospects for the synthesis of various keto acids [28]. Additionally, the variants of PmiLAAD have potential in the production of different kinds of D-amino acids when constructing multi-enzyme systems with other catalysts such as D-amino acid dehydrogenase [19,20], ammonia lyase [18], and D-amino acid transferase [21].

\subsection{Structural Analysis of E417 and Its Mutations}

The structural analysis showed that residue E417 is located in the loop structure of the substrate entrance channel of PmiLAAD (Figure S2), and is thus expected to play an important role in substrate entry and product release [28].

The enzyme activity measurements suggested that the ability to attain activity improvement is complex; however, we can identify two general features required. The first is that mutation on residue E417 to basic amino acid residues (such as E417K) universally improved the catalytic efficiency on the substrates and broadened the substrate spectrum. The other feature is that the most significant activity enhancement was achieved for the bulky substrate L-Trp. 
For example, when site 417 was mutated to Leu, the activity improvement was the highest (8.44-fold of the wild-type). It was speculated that E417L effectively expanded the substrate channel and alleviated the steric hindrance of the bulky substrate entering the pocket to improve the catalytic efficiency (Figure 5B). However, the mutant E417A was not as effective as E417L, with the activity increasing by only 2.73 times. This was probably attributed to the even larger substrate channel of PmiLAAD ${ }^{\mathrm{E} 417 \mathrm{~A}}$, which improved the degree of freedom of the entry of the substrate (Figure 5C), and the result of catalytic activity suggested that too much freedom of substrate entry would have a negative impact on the reaction efficiency [29]. Therefore, we measured the kinetic parameters (Table 2, Figure S4) of wild-type PmiLAAD and the variants PmiLAAD ${ }^{\mathrm{E} 417 \mathrm{~A}}$ and PmiLAAD ${ }^{\mathrm{E} 417 \mathrm{~L}}$ and found that the mutation of the entrance channel had little effect on the affinity of the substrate $\left(K_{\mathrm{m}}\right)$, whereas $k_{\text {cat }}$ was increased by mutants E417L and E417A. Thus, we speculated that moderate and proper expansion of the channel could significantly improve the catalytic efficiency of the enzyme. The $V_{\max }$ of the optimal mutant E417L was 4.8 -fold that of wild-type PmiLAAD. We also noticed that the activity of PmiLAAD ${ }^{\mathrm{E} 417 \mathrm{I}}$ towards L-Trp was decreased and the size of Ile is between Val and Leu. This may be attributed to the different position and orientation of the methyl group in the side chain of Ile, which leads to different molecular interactions in space and may result in decreased activity. In addition, the catalytic activity may not only be influenced by the size but also the shape of the entrance tunnel.

Table 2. The apparent kinetic parameters of PmiLAAD variants and the wild-type (WT) ${ }^{\text {a }}$.

\begin{tabular}{|c|c|c|c|c|c|}
\hline Substrate & Biocatalyst & $K_{\mathrm{m}}(\mathrm{mM})$ & $\left.V_{\max }\left(\mu \mathrm{M} \cdot \mathrm{mg}^{-1} \cdot \min ^{-1}\right)\right)$ & $k_{\text {cat }}\left(\mathrm{s}^{-1}\right)$ & $k_{\mathrm{cat}} / K_{\mathrm{m}}\left(\mathrm{mM}^{-1} \cdot \mathrm{s}^{-1}\right)$ \\
\hline \multirow{3}{*}{ L-Trp } & E417A & $7.92 \pm 1.59$ & $3.25 \pm 0.14$ & $2.78 \pm 0.12$ & 0.35 \\
\hline & E417L & $7.33 \pm 1.76$ & $8.53 \pm 0.36$ & $7.31 \pm 0.31$ & 1.00 \\
\hline & WT & $6.01 \pm 0.92$ & $1.76 \pm 0.05$ & $1.51 \pm 0.04$ & 0.25 \\
\hline \multirow{2}{*}{ L-Asp } & E417K & $11.61 \pm 2.11$ & $0.94 \pm 0.06$ & $0.81 \pm 0.05$ & 0.07 \\
\hline & WT & $21.88 \pm 5.20$ & $1.17 \pm 0.13$ & $1.00 \pm 0.11$ & 0.05 \\
\hline
\end{tabular}

a Note: The method of kinetic analysis using whole-cell biocatalysts referred to the method reported by Hossain et al. [30]. The reaction mixture consisted of different concentrations of L-amino acids and whole-cell biocatalysts of PmiLAAD variants. All experiments were conducted at $30^{\circ} \mathrm{C}$ in $50 \mathrm{mM}$ Tris- $\mathrm{HCl}$ buffer (pH 9.0) The amount of whole-cell biocatalyst in the reaction mixture was equal in quantity. The values were averaged from triplicate measurements.

A

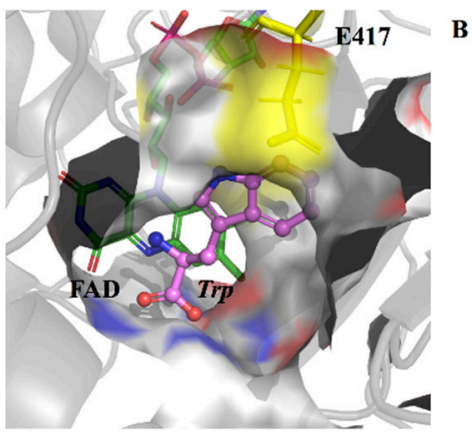

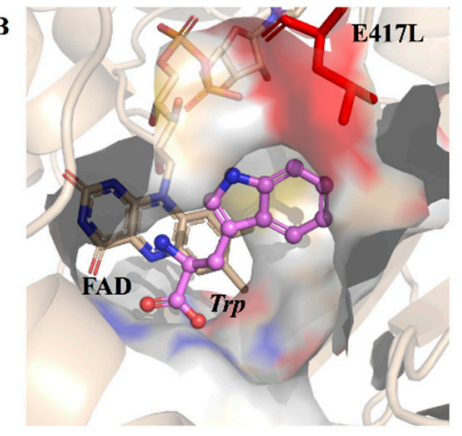

Figure 5. The substrate entrance channel was expanded by the mutations E417L and E417A. (A) The substrate entrance channel of wild-type PmiLAAD. The residue E417 is shown in yellow sticks. (B) The substrate entrance channel of PmiLAAD ${ }^{\mathrm{E} 417 \mathrm{~L}}$. The Leu residue on site 417 is shown in red sticks. (C) The substrate entrance channel of PmiLAAD ${ }^{\mathrm{E} 417 \mathrm{~A}}$. The Ala residue on site 417 is shown in magenta sticks.

The heatmaps in Figure 4 show that the catalytic efficiency of mutations E417H, E417K, and E417R (basic amino acids with larger side chains) towards L -Trp was increased, and these mutations to basic amino acids also generally improved the activity toward various $\mathrm{L}$ -amino acids. This is likely attributed to the basic side chain residue at site 417 , which had 
an affinity with the carboxyl group of the L-amino acid substrate. The kinetic parameters (Table 2) showed that the $K_{\mathrm{m}}$ of PmiLAAD ${ }^{\mathrm{E} 417 \mathrm{~K}}$ to L -Asp was significantly decreased compared to that of the wild-type, which confirmed that the affinity between the variant and the substrate was increased. This suggests that the catalytic efficiency of the enzyme was affected by not only the size of the entrance channel residues, but also the electrostatic condition of the channel. The influence of charged side chains on the substrate channel requires further study.

\section{Materials and Methods}

\subsection{Materials}

The host strain E. coli BL21 (DE3) harboring the expression plasmid pET-28a and the PmiLAAD gene from P. mirabilis (GenBank accession no. EU669819.1) were stored in our laboratory [20]. The DNA purification kit, PrimeSTAR ${ }^{\circledR}$ HS DNA polymerase premix, a one-step cloning kit, NdeI, and other reagents were acquired from Takara Bio Co. (Kawasaki, Japan) and Vazyme Biotech Co., Ltd. (Nanjing, China). L-Amino acids were purchased from Aladdin Biochemical Technology Co., Ltd. (Shanghai, China) and Sinopharm Chemical Reagent Co., Ltd. (Shanghai, China). Keto acids were purchased from Sigma-Aldrich (St. Louis, MO, USA). DNPH used for high-throughput screening was purchased from Sinopharm Chemical Reagent Co., Ltd. Sulfuric acid for high-performance liquid chromatography (HPLC) was purchased from Sinopharm Chemical Reagent Co., Ltd.

\subsection{Molecular Modeling and Docking Calculations}

The homologous structural models of PmiLAAD and its mutants were constructed using the SWISS-MODEL online server (https:/ / swissmodel.expasy.org/interactive, accessed on 1 January 2022), based on the X-ray crystal structures of PvLAAD with 91.93\% identity (PDB ID: 5hxw, GenBank accession no. AB030003.1). The structures of L-Ala, L-Asp, L-Ile, L-Leu, L-Phe, L-Trp, and L-Val were downloaded from PubChem (https: / / pubchem.ncbi.nlm.nih.gov, accessed on 1 January 2022). The seven L-amino acids were docked to the PmiLAAD receptor using AutoDock. The rank of docking results for each ligand was listed in Table S2. Analysis of the structures and identification of potential binding sites were performed using PyMOL.

\subsection{Alanine Scanning and Site-Directed Saturation Mutagenesis}

The residues within a $4 \AA$ scope around the substrate-binding site were selected for alanine scanning, and three key residues with higher activity were determined for site-directed saturation mutagenesis. Site-directed saturation mutants were constructed by homologous recombination according to a previously published method [31]. Primer synthesis and DNA sequencing were performed by Genewiz Biotech Co., Ltd. (Suzhou, China). The primers used in this study are listed in Table S1.

\subsection{Cell Culture and Protein Expression of PmiLAAD Mutants}

The site-directed saturation mutants of PmiLAAD expressed in E. coli BL21(DE3) were inoculated into 48 -well plates with $2 \mathrm{~mL}$ of Luria-Bertani medium containing $5 \mathrm{mg} \cdot \mathrm{mL}^{-1}$ kanamycin per well, and cultured at $37^{\circ} \mathrm{C}$. The culture was induced by the addition of isopropyl $\beta$-D-1-thiogalactopyranoside to a final concentration of $0.5 \mathrm{mM}$ when the optical density at $600 \mathrm{~nm}$ was $0.6-0.8$, followed by incubation for an additional $16 \mathrm{~h}$ at $17{ }^{\circ} \mathrm{C}$ with shaking at $200 \mathrm{rpm}$. The mutants were harvested by centrifugation at $4500 \mathrm{rpm}$ and $4{ }^{\circ} \mathrm{C}$ for $20 \mathrm{~min}$, and the mutant PmiLAAD whole cells were used for further activity screening.

\subsection{Measurement of Enzymatic Activity}

The whole-cell catalysts in the 48 -well plates obtained by centrifugation (as described above) were incubated with $2 \mathrm{~mL}$ of $50 \mathrm{mM}$ L-amino acid solution at $30^{\circ} \mathrm{C}$ with shaking at $200 \mathrm{rpm}$ for the oxidative reaction. The reaction mixture was centrifuged (4500 rpm, $20 \mathrm{~min}$ ) at $1 \mathrm{~h}, 3 \mathrm{~h}$, and $5 \mathrm{~h}$, and $100 \mu \mathrm{L}$ of the supernatant was transferred to a 96-well plate to 
measure keto acids. The biocatalytic activity of the PmiLAAD mutants was measured using the DNPH method reported by Zhou et al. [32]. Briefly, $50 \mu \mathrm{L}$ DNPH (2 mM, dissolved in $1.2 \mathrm{mM}$ diluted sulfuric acid) was added to $100 \mu \mathrm{L}$ of the above-mentioned samples and incubated at $30{ }^{\circ} \mathrm{C}$ for $5 \mathrm{~min}$. Then, $50 \mu \mathrm{L} \mathrm{NaOH}(4 \mathrm{M})$ was added, and the absorbance of the reddish-brown solution in the plates was measured at the corresponding wavelength of each keto acid using an absorbance microplate reader (CYTATION3, BioTek, Winooski, VT, US). The wavelength for the concentration measurement of each keto acid was determined by full-wavelength scanning. The wavelength with maximum absorbance was determined as the detection wavelength. The standard curves of the seven types of keto acids with different concentrations were obtained using the same DNPH-based method. One unit of PmiLAAD catalytic activity was defined as $1 \mu \mathrm{mol}$ of keto acid generated per minute under the assay conditions. All experiments were conducted in triplicate.

\subsection{Determination of Kinetic Parameters}

The method of kinetic analysis using whole-cell biocatalysts was reported by Hossain et al. [30]. Kinetic analysis of keto acid production by PmiLAAD mutants was conducted by measuring the concentration of keto acids produced with different concentrations of L-amino acids at $30{ }^{\circ} \mathrm{C}$ for $40 \mathrm{~min}$. The concentrations of L-Asp were $0-50 \mathrm{mM}$, and the concentrations of L-Trp were $0-80 \mathrm{mM}$. L-amino acids and whole-cell biocatalysts of PmiLAAD variants were mixed in $50 \mathrm{mM}$ Tris- $\mathrm{HCl}$ buffer ( $\mathrm{pH}$ 9.0). The amount of whole-cell biocatalyst in the reaction mixture was equal in quantity. The apparent $K_{\mathrm{m}}$ and $k_{\text {cat }}$ values were then calculated using the Michaelis-Menten equation. The values were averaged from triplicate measurements.

\subsection{Analysis of Produced Keto Acids}

The reaction samples $(100 \mu \mathrm{L})$ were filtered through a $0.45 \mu \mathrm{m}$ pore filter membrane (Organo system), and the resulting filtrate samples were tested using HPLC (Chromaster CM5110, Hitachi, Tokyo, Japan) with an Aminex HPX-87H column $(300 \times 7.8 \mathrm{~mm}, 9 \mu \mathrm{m})$. The mobile phase was $5 \mathrm{mM}$ sulfuric acid $\left(\mathrm{H}_{2} \mathrm{SO}_{4}\right)$ at a constant flow rate of $0.6 \mathrm{~mL} \cdot \mathrm{min}^{-1}$ $\left(40{ }^{\circ} \mathrm{C}\right)$. The injection volume was $10 \mu \mathrm{L}$, and the injection time was $30 \mathrm{~min}$. The ultraviolet detection wavelength was $210 \mathrm{~nm}$. The retention time of each keto acid is listed in Table S3. The standard curves of keto acids obtained by HPLC were compared with those obtained by the DNPH method, and the feasibility and validity of the DNPH method were confirmed.

\section{Conclusions}

In this study, we employed a semi-rational design strategy to broaden the substrate spectrum of PmiLAAD. The molecular docking method was used to predict the residues around the substrate-binding sites, and the alanine-scanning strategy was employed to determine the key residues for site-directed saturation. By site-directed saturation mutagenesis, site E417 was identified as a key site for catalytic efficiency improvement. The catalytic efficiency improvement obtained by a mutation on E417 may be attributed to the expansion of the substrate channel and its electrostatic condition. Expansion of the substrate channel (such as via the E417L mutation) improved the activity toward the bulky substrate L-Trp, and the mutation of E417 to basic amino acids (such as E417K) had a universal enhanced activity towards various L-amino acids. The substrate specificity of the PmiLAAD wild-type is mostly concentrated on several bulky or basic L-amino acids such as L-Phe, L-Leu, and L-His. In addition to these, the PmiLAAD variants in our study exhibited improved activity toward various kinds of L-amino acids, including non-polar L-amino acids (e.g., L-Trp and L-Val) and acidic L-amino acids (e.g., L-Asp). Consequently, the substrate spectrum of PmiLAAD was broadened on some level. Our study helps to expand the catalytic ability of PmiLAAD from a new perspective and provides a great candidate site, E417, to increase the substrate specificity of PmiLAAD. These PmiLAAD variants with broadened substrate specificity can not only be used for synthesizing keto 
acids, but they also have the potential to be applied in multi-enzyme cascade systems with other catalysts for producing D-amino acids.

Supplementary Materials: The following supporting information can be downloaded at: https: / / www.mdpi.com/article/10.3390/catal12020175/s1, Figure S1: The results of alanine scanning; Figure S2: Structure of PmiLAAD; Figure S3: Relative activity of PmLAAD ${ }^{\text {417K }}$ (A), PmLAAD ${ }^{\text {E417H }}$ (B), and PmLAAD ${ }^{\mathrm{E} 417 \mathrm{R}}$ (C) toward seven kinds of L-amino acids; Figure S4: Plots for the apparent kinetic parameter determination of PmiLAAD variants and wild-type; Table S1: Primers used in this study; Table S2: The rank of docking results of each ligand; Table S3: The retention times of high-performance liquid chromatography (HPLC).

Author Contributions: Conceptualization, A.F., Y.N. and Y.X.; methodology, A.F.; investigation, A.F., Z.W. and H.Q.; formal analysis, A.F. and Z.W.; validation, A.F., Z.W. and H.Q.; writing-original draft preparation, A.F.; writing - review and editing, Y.N.; supervision, Y.N. and Y.X.; funding acquisition, Y.N. All authors have read and agreed to the published version of the manuscript.

Funding: This work was financially supported by the National Key R\&D Program of China (grant number 2021YFC2102000), the National Natural Science Foundation of China (grant numbers 22178147, 31872891), the Program of Introducing Talents of Discipline to Universities (grant number 111-2-06), the High-End Foreign Experts Recruitment Program (grant number G2021144005L), the Program for Advanced Talents within Six Industries of Jiangsu Province (grant number 2015-NY-007), the National Program for Support of Top-notch Young Professionals, the Project Funded by the Priority Academic Program Development of Jiangsu Higher Education Institutions, the Top-notch Academic Programs Project of Jiangsu Higher Education Institutions, the Jiangsu province "Collaborative Innovation Center for Advanced Industrial Fermentation" industry development program, and the National First-Class Discipline Program of Light Industry Technology and Engineering (grant number LITE2018-09).

Conflicts of Interest: The authors declare no conflict of interest.

\section{References}

1. Motta, P.; Molla, G.; Pollegioni, L.; Nardini, M. Structure-Function Relationships in 1-Amino Acid Deaminase, a Flavoprotein Belonging to a Novel Class of Biotechnologically Relevant Enzymes. J. Biol. Chem. 2016, 291, 10457-10475. [CrossRef] [PubMed]

2. Molla, G.; Melis, R.; Pollegioni, L. Breaking the mirror: L-Amino acid deaminase, a novel stereoselective biocatalyst. Biotechnol. Adv. 2017, 35, 657-668. [CrossRef] [PubMed]

3. Song, Y.; Li, J.; Shin, H.-D.; Liu, L.; Du, G.; Chen, J. Biotechnological production of alpha-keto acids: Current status and perspectives. Bioresour. Technol. 2016, 219, 716-724. [CrossRef] [PubMed]

4. Okino, S.; Suda, M.; Fujikura, K.; Inui, M.; Yukawa, H. Production of d-lactic acid by Corynebacterium glutamicum under oxygen deprivation. Appl. Microbiol. Biotechnol. 2008, 78, 449-454. [CrossRef] [PubMed]

5. Gao, X.; Ma, Q.; Zhu, H. Distribution, industrial applications, and enzymatic synthesis of d-amino acids. Appl. Microbiol. Biotechnol. 2015, 99, 3341-3349. [CrossRef] [PubMed]

6. Li, X.; Jiang, B.; Pan, B. Biotransformation of phenylpyruvic acid to phenyllactic acid by growing and resting cells of a Lactobacillus sp. Biotechnol. Lett. 2007, 29, 593-597. [CrossRef]

7. Subhi, A.L.; Diegelman, P.; Porter, C.W.; Tang, B.; Lu, Z.J.; Markham, G.D.; Kruger, W.D. Methylthioadenosine Phosphorylase Regulates Ornithine Decarboxylase by Production of Downstream Metabolites. J. Biol. Chem. 2003, 278, 49868-49873. [CrossRef]

8. Dilger, R.; Kobler, C.; Weckbecker, C.; Hoehler, D.; Baker, D.H. 2-Keto-4-(Methylthio)Butyric Acid (Keto Analog of Methionine) Is a Safe and Efficacious Precursor of 1-Methionine in Chicks. J. Nutr. 2007, 137, 1868-1873. [CrossRef]

9. Ichikawa, T.; Maeda, S.; Araki, Y.; Ishido, Y. Synthetic studies of amino acids by the use of the copper complex. I. Syntheses of. beta.-hydroxy DL-amino acids by the use of the complex of copper(II) with the Schiff base derived from glycine and pyruvic acid. J. Am. Chem. Soc. 1970, 92, 5514-5516. [CrossRef]

10. Kerber, R.C.; Fernando, M.S. $\alpha$-Oxocarboxylic Acids. J. Chem. Educ. 2010, 87, 1079-1084. [CrossRef]

11. Escobar, J.; Frank, J.W.; Suryawan, A.; Nguyen, H.V.; Van Horn, C.G.; Hutson, S.M.; Davis, T.A. Leucine and $\alpha$-Ketoisocaproic Acid, but Not Norleucine, Stimulate Skeletal Muscle Protein Synthesis in Neonatal Pigs. J. Nutr. 2010, 140, 1418-1424. [CrossRef]

12. Mitch, W.E. Dietary therapy in uremia: The impact on nutrition and progressive renal failure. Kidney Int. 2000, 57, S38-S43. [CrossRef]

13. Wang, X.; Hou, Y.; Liu, L.; Li, J.; Du, G.; Chen, J.; Wang, M. A new approach for efficient synthesis of phenyllactic acid from L-phenylalanine: Pathway design and cofactor engineering. J. Food Biochem. 2018, 42, e12584. [CrossRef]

14. Ding, H.; Zhao, W.; Lü, C.; Huang, J.; Hu, S.; Yao, S.; Mei, L.; Wang, J.; Mei, J. Biosynthesis of 4-hydroxyphenylpyruvic acid from L-tyrosine using recombinant Escherichia coli cells expressing membrane bound L-amino acid deaminase. Chin. J. Chem. Eng. 2018, 26, 380-385. [CrossRef] 
15. Hossain, G.S.; Li, J.; Shin, H.-D.; Du, G.; Wang, M.; Liu, L.; Chen, J. One-Step Biosynthesis of $\alpha$-Keto- $\gamma$-Methylthiobutyric Acid from L-Methionine by an Escherichia coli Whole-Cell Biocatalyst Expressing an Engineered L-Amino Acid Deaminase from Proteus vulgaris. PLoS ONE 2014, 9, e114291. [CrossRef] [PubMed]

16. Friedman, M. Origin, Microbiology, Nutrition, and Pharmacology of D-Amino Acids. Chem. Biodivers. 2010, 7, 1491-1530. [CrossRef]

17. Martínez-Rodríguez, S.; Martínez-Gómez, A.I.; Rodríguez-Vico, F.; Clemente-Jiménez, J.M.; Las Hera-Vázquez, F.J. Natural Occurrence and Industrial Applications of D-Amino Acids: An Overview. Chem. Biodivers. 2010, 7, 1531-1548. [CrossRef]

18. Parmeggiani, F.; Lovelock, S.L.; Weise, N.J.; Ahmed, S.T.; Turner, N.J. Synthesis ofD- andL-Phenylalanine Derivatives by Phenylalanine Ammonia Lyases: A Multienzymatic Cascade Process. Angew. Chem. Int. Ed. 2015, 54, 4608-4611. [CrossRef]

19. Parmeggiani, F.; Ahmed, S.T.; Thompson, M.P.; Weise, N.J.; Galman, J.; Gahloth, D.; Dunstan, M.S.; Leys, D.; Turner, N.J. Single-Biocatalyst Synthesis of Enantiopured-Arylalanines Exploiting an Engineeredd-Amino Acid Dehydrogenase. Adv. Synth. Catal. 2016, 358, 3298-3306. [CrossRef]

20. Zhang, D.; Jing, X.; Zhang, W.; Nie, Y.; Xu, Y. Highly selective synthesis of d-amino acids from readily available L-amino acids by a one-pot biocatalytic stereoinversion cascade. RSC Adv. 2019, 9, 29927-29935. [CrossRef]

21. Walton, C.J.W.; Parmeggiani, F.; Barber, J.E.B.; McCann, J.L.; Turner, N.J.; Chica, R.A. Engineered Aminotransferase for the Production of $d$-Phenylalanine Derivatives Using Biocatalytic Cascades. ChemCatChem 2018, 10, 470-474. [CrossRef]

22. Wu, L.; Guo, X.; Wu, G.; Liu, P.; Liu, Z. Efficient enzymatic synthesis of $\alpha$-keto acids by redesigned substrate-binding pocket of the L-amino acid deaminase (PmiLAAD). Enzym. Microb. Technol. 2020, 132, 109393. [CrossRef] [PubMed]

23. Song, Y.; Li, J.; Shin, H.-D.; Liu, L.; Du, G.; Guocheng, D. Tuning the transcription and translation of L-amino acid deaminase in Escherichia coli improves $\alpha$-ketoisocaproate production from L-leucine. PLoS ONE 2017, 12, e0179229. [CrossRef] [PubMed]

24. Li, R.; Sakir, H.G.; Li, J.; Shin, H.-D.; Du, G.; Chen, J.; Liu, L. Rational molecular engineering of L-amino acid deaminase for production of $\alpha$-ketoisovaleric acid from L-valine by Escherichia coli. RSC Adv. 2017, 7, 6615-6621. [CrossRef]

25. Melis, R.; Rosini, E.; Pirillo, V.; Pollegioni, L.; Molla, G. In vitro evolution of an L-amino acid deaminase active on L-1naphthylalanine. Catal. Sci. Technol. 2018, 8, 5359-5367. [CrossRef]

26. Ju, Y.; Tong, S.; Gao, Y.; Zhao, W.; Liu, Q.; Gu, Q.; Xu, J.; Niu, L.; Teng, M.; Zhou, H. Crystal structure of a membrane-bound L-amino acid deaminase from Proteus vulgaris. J. Struct. Biol. 2016, 195, 306-315. [CrossRef] [PubMed]

27. Morrison, K.L.; Weiss, G.A. Combinatorial alanine-scanning. Curr. Opin. Chem. Biol. 2001, 5, 302-307. [CrossRef]

28. Wu, Y.; Zhang, S.; Song, W.; Liu, J.; Chen, X.; Hu, G.; Zhou, Y.; Liu, L.; Wu, J. Enhanced Catalytic Efficiency of L-amino Acid Deaminase Achieved by a Shorter Hydride Transfer Distance. ChemCatChem 2021, 13, 4557-4566. [CrossRef]

29. Li, G.; Yao, P.; Gong, R.; Li, J.; Liu, P.; Lonsdale, R.; Wu, Q.; Lin, J.; Zhu, D.; Reetz, M.T. Simultaneous engineering of an enzyme's entrance tunnel and active site: The case of monoamine oxidase MAO-N. Chem. Sci. 2017, 8, 4093-4099. [CrossRef]

30. Hossain, G.S.; Li, J.; Shin, H.D.; Liu, L.; Wang, M.; Du, G.; Chen, J. Improved production of alpha-ketoglutaric acid (alpha-KG) by a Bacillus subtilis whole-cell biocatalyst via engineering of L-amino acid deaminase and deletion of the alpha-KG utilization pathway. J. Biotechnol. 2014, 187, 71-77. [CrossRef]

31. Wang, X.; Nie, Y.; Xu, Y. Improvement of the Activity and Stability of Starch-Debranching Pullulanase from Bacillus naganoensis via Tailoring of the Active Sites Lining the Catalytic Pocket. J. Agric. Food Chem. 2018, 66, 13236-13242. [CrossRef] [PubMed]

32. Zhou, J.; Xu, G.; Han, R.; Dong, J.; Zhang, W.; Zhang, R.; Ni, Y. Carbonyl group-dependent high-throughput screening and enzymatic characterization of diaromatic ketone reductase. Catal. Sci. Technol. 2016, 6, 6320-6327. [CrossRef] 